\title{
Commentary
}

\section{Host-manipulation by parasites: towards a neuroethological approach?}

\author{
Frank Cézilly \\ F. Cézilly (frank.cezilly@u-bourgogne.fr), Université de Bourgogne; Equipe Ecologie Evolutive, UMR CNRS 5561 \\ Biogéosciences
}

In several host-parasite systems, infected hosts show phenotypic alterations that reduce their own fitness but appear to increase that of the parasite. Although, the ecological aspects of host manipulation have received large attention, little is known about the mechanisms by which parasites succeed in taking control of their host. Salwiczek and Wickler (2009) suggest, however, that unravelling the mechanistic basis of manipulation would not help much in understanding how parasites induce phenotypic alterations in their host. They recommend instead a "thorough ethological approach" to host manipulation. Their main argument is that the parasites are unable to induce a novel behaviour in their hosts, but instead exploit part of the behavioural repertoire of the host species which is usually unexpressed in uninfected hosts. This idea is indeed important, although not totally new (see for instance Eberhard 2000), and certainly deserves further consideration. However, in my opinion, Salwiczek and Wickler (2009) go too far in their claim that an ethological approach provides an alternative to mechanistic accounts of host manipulation. I will argue here that actually the two approaches should be integrated in a more general approach to host manipulation by parasites.

Part of the issue raised by the authors is clearly a matter of semantics. Salwiczek and Wickler (2009) correctly point out that several researchers working on host manipulation have used the terms "aberrant", "new" or "novel" to qualify the phenotypic alterations observed in infected hosts. However, my feeling is that those terms are generally employed simply to mean "not usually observed in uninfected hosts". Any reasonable evolutionary ecologist would accept that a parasite can only modify or alter what is already present in its host. In that sense, paraphrasing François Jacob (1977), host manipulation could be seen as tinkering.
There are, however, several weaknesses in the argumentation developed by Salwiczek and Wickler (2009). First, a large number of phenotypic alterations observed in infected hosts simply consist of reversed taxis (moves to/away from stimulus), such as phototaxis or geotaxis. For instance, amphipods infected with the acanthocephalan parasites of the genus Pomphorhynchus are attracted to light, whereas uninfected individuals show typically negative phototaxis (Cézilly et al. 2000, Perrot-Minnot et al. 2007). Ample evidence exists that reversed phototaxis enhances trophic transmission of the parasites to appropriate final hosts (Perrot-Minnot et al. 2007, Kaldonski et al. 2009). Although the mechanisms by which parasitic infection leads to reversed phototaxis have not yet been identified, there is some evidence that reversed phototaxis is associated with an alteration of serotonergic (5-HT) activity in the brain of infected individuals (Tain et al. 2006, 2007). The problem here is not so much to decide whether reversed phototaxis must be regarded as a "novel" behaviour, or just as the mere alteration of a pre-existing behaviour. Whatever the answer to that question, understating how reversal is achieved remains a central question that is unanswered by a purely ethological approach.

Second, it seems that Salwiczek and Wickler (2009) have not fully understood the importance of multidimensionality in host manipulation (Cézilly and Perrot-Minnot 2005, Benesh et al. 2008). Multidimensionality does not necessarily correspond to a sequence of behaviours, as in the case of ants infected with Dicrocelium, but rather refers to the fact that infected hosts often show several simultaneous phenotypic alterations. For instance, isopods infected with the acanthocephalan Acanthocephalus anguillae show reversed phototaxis and a darkened cuticle. One 
important question is to understand whether these two phenotypic alterations are linked or independent from a mechanistic point of view.

Actually, the ideas developed by Salwiczek and Wickler (2009) are a strong argument in favour of the development of mechanistic approaches to host manipulation. Indeed, in most, if not all, of the cases they review, the mechanisms by which a dormant behaviour is reactivated remain to be elucidated. Why is this important? Because a key question in understanding the evolution of host-manipulation is to know whether manipulation must be regarded as an adaptation or as just a by-product of infection. One way to establish the adaptive character of manipulation would be to show that manipulative parasites have evolved an ability to reactivate some dormant behaviour in their hosts, beyond what would spontaneously result from the interference between the immune response of the host and its nervous system (see Adamo 2002). This could be achieved through comparing the mechanisms of manipulation between closely-related host-parasite systems that differ in the nature or intensity of manipulation. So far, unfortunately, data on the mechanisms of manipulation are too scarce to allow for such a comparative analysis.

To conclude, I concur with Salwiczek and Wickler (2009) that the study of host manipulation by parasites is a very promising avenue for behavioural research. Indeed, elucidating how parasites can influence gene expression and/or regulate some key neuromodulators in their hosts can potentially bring important insights about the evolution of behaviour. However, if a deep ethological analysis of host manipulation is welcome, it should not invalidate other approaches. Actually, a comparative neuroethological approach to host manipulation certainly seems most appropriate.

\section{References}

Adamo, S.A. 2002. Modulating the modulators: parasites, neuromodulators and host behavioural changes. Brain, Behavior and Evolution 60: 370377. CrossRef

Benesh, D. P., Valtonen, E. T. and O. Seppälä. 2008. Multidimensionality and intra-individual variation in host manipulation by an acanthocephalan. Parasitology 135: 617-626. CrossRef

Cézilly, F. and M.-J. Perrot-Minnot. 2005. Studying adaptive changes in the behaviour of infected hosts: a long and winding road. Behavioural Processes 68: 223-228. CrossRef

Cézilly, F., Grégoire, A. and A. Bertin. 2000. Conflict between co-occurring manipulative parasites? An experimental study of the joint influence of two acanthocephalan parasites on the behaviour of
Gammarus pulex. Parasitology 120: 625-630. CrossRef

Eberhard, W.G. 2000. Spider manipulation by a wasp larva. Nature 406: 255-256. CrossRef

Jacob, F. 1977. Evolution and tinkering. Science 196: 1161-1166. CrossRef

Perrot-Minnot, M.-J., Kaldonski, N. and F. Cézilly. 2007. Increased susceptibility to predation and altered anti-predator behaviour in an acanthocephalan-infected amphipod. International Journal for Parasitology 37: 645-651. CrossRef

Kaldonski, N., Perrot-Minnot, M.-J, Dodet, R., Martinaud, G. and F. Cézilly. 2009. Carotenoidbased colour of acanthocephalan cystacanths plays no role in host manipulation. Proceedings of the Royal Society Series B Biological Sciences 276: 169-176. CrossRef

Salwiczek, L.H. and W. Wickler. 2009. Parasites as scouts in behaviour research. Ideas in Ecology and Evolution 2: 1-6. CrossRef

Tain, L., Perrot-Minnot, M.-J. and F. Cézilly. 2006. Altered host behaviour and brain serotonergic activity caused by acanthocephalans: evidence for specificity. Proceedings of the Royal Society Series B Biological Sciences 273: 3039-3045. CrossRef

Tain, L., Perrot-Minnot, M.-J. and F. Cézilly. 2007. Differential influence of Pomphorhynchus laevis (Acanthocephala) on brain serotonergic activity in two congeneric host species. Biology Letters 3: 6871. CrossRef 